\title{
Österreich: Fremdbestimmung und Selbstzerstörung
}

Die lange Suche nach der - nach einer - Identität

Die Ausrufung der Republik Österreich am 12. November war 1918 eine ebenso pragmatische wie vernünftige Antwort auf eine geopolitische Gegebenheit. Am 3. November 1918 hatten die Streitkräfte Österreich-Ungarns kapituliert, am 11. November hatte der letzte österreichische Kaiser abgedankt. Schon am 21. Oktober hatten deutschsprachige Abgeordnete des 1911 gewählten österreichischen Reichsrats eine Provisorische Nationalversammlung gebildet. Es war diese, schon damals mit einem demokratischen Mandat ausgestattete Nationalversammlung, die den Beschluss zur Gründung einer Republik fasste - wenige Tage nachdem das Königreich Ungarn die Bindungen an die österreichische Reichshälfte der „Doppelmonarchie“ gelöst hatte, in Prag die Republik der Tschechen und Slowaken ausgerufen worden war, Triest von italienischen Truppen besetzt wurde und im österreichischen Galizien (Krakau, Lemberg) ein politisches Vakuum entstanden war.

\section{Zur Selbständigkeit gezwungen}

Am 12. November wurde eine Republik gegründet, die erst zur Selbstständigkeit gezwungen werden musste. Denn die Republik nannte sich zunächst „DeutschÖsterreich“ und erklärte, Teil der Deutschen Republik zu sein. Das aber widersprach den Plänen der Siegermächte, die jede Stärkung des zur Republik gewordenen Deutschen Reichs verhindern wollten. Der „Anschluss“ an Deutschland wurde Österreich von der Entente untersagt - und eben deshalb blieb der „Anschluss“ der Begriff, der die nächsten zwei Jahrzehnte die Geschichte Österreichs wie ein Geburtstrauma begleiten sollte.

Diese Republik, die sich Österreich nennen musste, wusste nicht so recht, was sie eigentlich war. Sie war einer der Nachfolgestaaten des Reichs der Habsburger - wie die Tschechoslowakei und Ungarn, wie auch - partiell - Polen und Rumänien, Jugoslawien und Italien. Doch die Republik war mit einem Namen belastet, der sie in eine Kontinuität stellte, die sie nicht beanspruchte. Der Vertrag, den Karl Renner als Kanzler der Provisorischen Staatsregierung am 10. September 1920 in St. Germain unterzeichnete, war formell auch kein Friedens-, sondern ein Staatsvertrag. Die Regierung der Republik konnte und wollte nicht beanspruchen, in der Nachfolge der Regierung Österreich-Ungarns zu stehen.

In St. Germain wurde Österreich geografisch definiert - als der Rest der Doppelmonarchie, der nicht von den anderen Nachfolgestaaten in Anspruch genommen werden konnte. Auf „Selbstbestimmung“ wurde von Seiten der Siegermächte keine Rücksicht genommen: So wurden der Republik Österreich keine der Gebiete zugesprochen, die mit Berufung auf das Selbstbestimmungsrecht von ihr beansprucht wurden - die mehrheitlich deutschsprachigen Gebiete Nordböhmens, 
Nordmährens und Südtirol. Das geopolitische Interesse der Sieger hatte Vorrang, Vorrang auch vor dem Interesse des kleinsten unter den Nachfolgestaaten des Habsburgerreichs, der sich - in der Tradition der Begrifflichkeit des „Vielvölkerstaats“ der Habsburger - als deutsch und damit als Teil eines republikanisch gewordenen Deutschen Reichs sehen wollte.

Die zur Selbstständigkeit gezwungene, fremdbestimmte Republik war dennoch in der Lage, sich eine - auch rückblickend gesehen - rationale und nachhaltige Grundlage zu geben: Am 1. Oktober 1920 beschloss die 1919 gewählte Konstituierende Nationalversammlung eine Verfassung, die nach fast einem Jahrhundert noch immer die Verfassung der Republik ist; mittlerweile die älteste geschriebene Verfassung eines europäischen Staats.

Die Gründer des nur widerwillig selbstständigen Staats waren durchwegs Männer, die aus dem Gestern kamen: Abgeordnete des 1911 nach dem allgemeinen und gleichen Männerwahlrecht gewählten Abgeordnetenhauses des Reichsrats. Sie waren zu Fraktionen zusammengeschlossen, zu Parteien, die sich bis 1918 als eine Art Reservemacht gebildet hatten - im alten Österreich, mit seiner vom Kaiser bestellten, dem Parlament des Kaiserreichs nicht verantwortlichen Regierung. Nun, nach dem Zusammenbruch der alten Ordnung einer konstitutionellen Monarchie und einem unterentwickelten Parlamentarismus, war diese Reservemacht im Zentrum des Geschehens. Es waren die Parteien, die mehr oder weniger gezwungen waren, aus dem, was vom alten Österreich übrig war, einen neuen Staat aufzubauen.

\section{Republik - aber welche?}

Die Republik begann als ein Parteienstaat. Die Sozialdemokratische Arbeiterpartei Österreichs (SDAP), die Christlichsoziale Partei (CSP) und die Deutschnationalen (die sich bald in Form von zwei kleinen Parteien, der Großdeutschen Volkspartei und dem Landbund, konstituieren sollten) hatten die Provisorische Regierung gebildet und in der Konstituierenden Nationalversammlung (bei deren Wahl auch erstmals das Wahlrecht für Frauen gewährleistet war) bestimmt, wie dieser „Staat wider Willen“ gestaltet werden sollte: als Republik, die dem (zunächst nur indirekt gewählten Staatsoberhaupt, dem Bundespräsidenten) nur eingeschränkt Machtbefugnisse zuordnete; als Bundesstaat, in dem neun Länder und der Bund sich die Macht teilten; als parlamentarisches System, das die Mehrheitsverhältnisse in dem direkt gewählten Nationalrat darüber entscheiden ließ, wer als „Bundesregierung“ über die Exekutivgewalt verfügte.

Diese in sich schlüssige Ordnung eines demokratischen Staatswesens sollte freilich bald die 1920 noch vorhandene breite politische Grundlage verlieren. Ab 1920 regierte ein „Bürgerblock“, eine Koalition aus CSP, Deutschnationalen und (ab 1930) den offen antidemokratischen „Heimwehren“. Die SDAP, die bei den 1920, 1923, 1927 und 1930 durchgeführten Parlamentswahlen immer etwa 40 Prozent der Stimmen gewinnen konnte, war in Daueropposition - und konzentrierte sich auf Wien, das zu einem Modell („Rotes Wien“) sozialdemokratischen Regierens gemacht werden sollte. 
Die Republik, die sich mit der von den Siegern des Weltkriegs vorgegebenen europäischen Ordnung notgedrungen arrangiert hatte, die sich eine demokratische Ordnung gegeben hatte - eine Ordnung, die eine lebendige Demokratie ermöglichte, diese Republik scheiterte. Sie konnte dem im mitteleuropäischen Raum zunehmend dominanten antidemokratischen Zeitgeist nicht widerstehen. Die Parteien, immer weniger von dem zwischen 1918 und 1920 herrschenden Basiskonsens bestimmt, entwickelten sich auseinander: Die Sozialdemokratie sah sich in ständigem Out und hoffte vergeblich auf den entscheidenden Durchbruch bei Wahlen. Sie tröstete sich mit einem „Austromarxismus“ genannten Überbau über ihre Isolierung hinweg, mit einer Ideologie, die den Sieg des „Sozialismus“ als letztlich unausweichlich betrachtete. Die CSP, fixiert auf die Soziallehre der katholischen Kirche, sah in der demokratischen Republik keinen Wert an sich, sondern eine Zwischenstufe auf dem Weg in eine „wahre Demokratie“, deren Konturen vage blieben. Und den Deutschnationalen kamen ab 1930 immer mehr ihre Wählerinnen und Wähler abhanden, die zu der auch in Österreich aufstrebenden NSDAP überliefen.

Am Ende stand die Ausschaltung des Parlaments durch eine zunehmend autoritäre Regierung unter dem Kanzler Engelbert Dollfuß (CSP), die sich auf das faschistische Italien stützte; stand der Bürgerkrieg vom 12. Februar 1934, in dem die Regierung den Widerstand der Sozialdemokratie mit militärischer Gewalt ausschaltete; stand ein am 1. Mai 1934 ausgerufener Bundesstaat, der als Ständestaat Elemente des italienischen Faschismus mit der Sozialdoktrin der katholischen Kirche in Einklang zu bringen versuchte; stand die Ermordung Dollfuß' durch nationalsozialistische Putschisten; stand der Zusammenbruch des halbfaschistischen Systems unter dem auch militärischen Druck des nationalsozialistischen Deutschland; stand das Ende Österreichs. Am Ende stand der „Anschluss“ - unter freilich anderen Vorzeichen als denen, die 1918 gegolten hätten.

Als die Republik scheiterte, schien ihr kaum jemand nachzutrauern. Die Sozialdemokraten im Untergrund und im Exil dachten über die Republik hinaus in Richtung einer vagen sozialistischen Ordnung; die CSP schwankte zwischen einer rückwärtsgewandten Orientierung am 1918 untergegangenen Österreich und einem semifaschistischen System, das den Segen der katholischen Kirche haben sollte; und die Deutschnationalen sahen sich - in ihrer großen Mehrheit - 1938 am Ziel ihrer Wünsche. Die Republik, sie schien erledigt, von der Geschichte überholt. Dass sie 1945 wiederkehren und eine stabile, auch sozial und wirtschaftlich erfolgreiche demokratische Ordnung ermöglichen sollte - das wäre 1933/34 ebenso kaum jemand in den Sinn gekommen wie auch 1938 das Wiedererstehen der Republik nicht realistisch zu sein schien.

\section{Volk - aber welches?}

Was die Demokratie bedeutete, wie sie in der Präambel der Verfassung von 1920 verkündet werden sollte, war umstritten; und umstritten war, wer und was das Volk war - das Volk, von dem, so die republikanische Verfassung, das Recht der Republik ausging. Im Sinne des Staatsgrundgesetzes von 1867, das von der Republik 
übernommen wurde, sollte niemand als Opfer ethnischer oder religiöser Diskriminierung vom Volk ausgeschlossen werden. Doch de facto herrschte im Alltag der Republik ein „völkisches“ Denken vor, das sich vor allem gegen eine Minderheit der Bürgerinnen und Bürger richtete: Jüdinnen und Juden wurden in verschiedenen Schattierungen von verschiedener Seite nicht als Teil des „Wir“, als Teil des „eigenen“ Volks gesehen. Offen und aggressiv vertraten die deutschnationalen Parteien antijüdische Positionen, und die CSP kam diesen Positionen auch programmatisch oft sehr nahe.

Der Antisemitismus hatte schon vor Gründung der Republik politisches Verhalten bestimmt, und es war nicht nur und auch nicht primär ein religiöses Verständnis, das zur Definition des Judentums herangezogen wurde: Jüdinnen und Juden konnten getauft sein oder auch nicht, sie wurden von der herrschenden Stimmung als nicht voll „dazu gehörig“ gesehen - eine Abgrenzung, die direkt zum Begriff einer erfundenen ,jüdischen Rasse“ führte.

Der herrschende Antisemitismus war dafür verantwortlich, dass sich aus der als jüdisch definierten Minderheit kaum Personen politisch engagierten, die sich in irgendeiner Form zum Judentum bekannten. In der SDAP, die in aller Form dem Antisemitismus eine Absage erteilte, spielten Personen mit jüdischen Wurzeln oft eine prominente Rolle - wie Otto Bauer oder Julius Deutsch. Aber diese Personen, die aus dem Judentum kamen, schienen sich für ihre Herkunft fast zu schämen. Sie verdrängten sie - und waren eben deshalb nicht in der Lage, den Antisemitismus zu bekämpfen.

Der Republik und ihren Parteien gelang es nicht, sich vom Bewusstseinsstand und der Begrifflichkeit des alten Österreich zu emanzipieren. Österreich, das war bis 1918 ein Oberbegriff, unter dem sich die verschiedenen Nationalitäten versammelt hatten - die deutsche und die tschechische, die polnische und die ruthenische (ukrainische), die italienische und die slowenische Nationalität. Anders als in der ungarischen „Reichshälfte“, die sich als ungarischer Nationalstaat mit zu tolerierenden Minderheiten verstand, war das alte Österreich in seinem offiziellen, 1867 formulierten Verständnis kein Nationalstaat, definiert über eine „Kernnation“. Daher verstand sich die 1918 gegründete Republik als Staat der deutschen Nationalität - und hatte, nachdem der in diesem Verständnis logisch angelegte „Anschluss“ an das Deutsche Reich zunächst nicht möglich war, wesentliche Probleme mit ihrem Selbstverständnis.

Die SDAP strich erst 1933 die Forderung nach dem „Anschluss“ an Deutschland aus ihrem Programm - als Deutschland bereits die Züge der NS-Diktatur angenommen hatte; und Bauer reagierte im Pariser Exil auf den im März 1938 durch den Einmarsch deutscher Truppen vollzogenen „Anschluss“ mit der Forderung nach einer „gesamtdeutschen Revolution“ - und einer ausdrücklichen Absage an die Wiederherstellung der Republik Österreich. Signifikant war auch, dass der autoritär regierende Kanzler, Kurt Schuschnigg, am 11. März in einer Rundfunkrede seine Kapitulation mit der Feststellung begründete, er wolle kein „deutsches Blut"vergießen und sich mit einem „deutschen Wort“ („Gott schütze Österreich“) verabschiedete. Mit so viel deutscher Gesinnung war es letztlich nicht möglich, 
sich diesem nunmehr von Adolf Hitler regierten Deutschland entgegen zu stellen.

\section{Demokratie - aber welche?}

Die Demokratie, wie sie der Verfassung von 1920 entsprach, war in ihrem Gerüst von Institutionen und Prozessen eine voll entwickelte parlamentarische Demokratie. Aber sie war eine Kopfgeburt. Die Akteure hatten sich zwischen 1918 und 1920 auf die Staatsform einer demokratischen Republik geeinigt, weil diese der kleinste gemeinsame Nenner war, auf den sich die „austromarxistische“ Sozialdemokratie, die dem politischen Katholizismus verpflichtete CSP und die mehr als alle anderen am „Anschluss“ orientierten deutschnationalen Parteien einigen konnten. Aber als eigentliches Ziel, als Primärwert, wurde diese demokratische Republik nicht gesehen.

Für die Sozialdemokratie, die sich mehr als die anderen mit der Republik identifizierte, war die Demokratie ein Sekundärwert - auf dem Weg zum Sozialismus, den sie in Abgrenzung von der kleinen Kommunistischen Partei Österreichs mit den Mitteln des Parlamentarismus erreichen wollten. Für die CSP verlor die Demokratie rasch an Wert, als der für diese Partei entscheidende Akteur, die katholische Kirche, sich zunehmend am autoritären System zu orientieren begann, ausgedrückt etwa 1929 in den Lateran-Verträgen zwischen dem „Heiligen Stuhl“ und dem faschistischen Italien. Und die Deutschnationalen - nicht alle, aber die meisten - waren rasch bereit, demokratische Grundsätze dem „Anschluss“ an ein totalitäres Deutsches Reich unterzuordnen.

Demokratie wurde als „Westminster Demokratie“ verstanden - aufbauend auf dem Grundsatz The Winner Takes It All. Diese auf dem Prinzip der Konkurrenz aufbauende Demokratie beruhte auch auf der Möglichkeit der Rotation zwischen Regierungs- und Oppositionsrolle. Aber eben das wurde nach 1920 nicht umgesetzt - die Sozialdemokratie blieb in permanenter Opposition. Der Grundgedanke eines Konsenses war ab 1920 immer mehr in den Hintergrund getreten. Der Wettbewerb der Parteien wurde immer mehr zu einer Auseinandersetzung zwischen einander ausschließenden „Weltanschauungen“.

Chancen, Demokratie nicht nur als Nullsummenspiel zu verstehen, waren vorhanden. Und sie wurden auch punktuell genutzt - insbesondere zwischen 1918 und 1920, als die Parteien eine in sich schlüssige, belastbare Verfassung erarbeiteten. Bald schon aber machte die Konsensbereitschaft einer manichäischen Sicht der Politik Platz: Die demokratische Republik wurde immer mehr als Kampfboden gesehen, auf dem die Kräfte des Lichts auf die Kräfte der Finsternis trafen. Der eigene Erfolg wurde primär als Niederlage der jeweils anderen Seite definiert.

Das sollte sich nach 1945 ändern. In ihrem zweiten Anlauf entwickelte die Republik Österreich eine politische Kultur der Konkordanz. Unbeschadet des Wettbewerbs der Parteien um Wählerstimmen beteiligten die beiden nach 1945 hegemonialen Parteien, die Sozialdemokratische Partei und die aus der Tradition der CSP kommende Österreichische Volkspartei, die jeweils andere Seite an der Ausübung politischer Macht: in Form von Koalitionsregierungen (zwischen 1945 und 
1966, 1987 und 2000, und wieder von 2007 bis 2017). Überdies sicherte ein dichtes Netzwerk einer Sozialpartnerschaft, an der sich Arbeitgeber- und Arbeitnehmer ebenso beteiligten wie die Landwirtschaft, eine Machtbalance. Am Fehlen eines solchen Gleichgewichts war die Republik in ihrem ersten Versuch gescheitert.

\section{Was blieb?}

1918 nahm die neu gegründete Republik Österreich eine besondere Stellung in ihrem geopolitischen Umfeld ein. Sie war ein Nachfolgestaat des alten Reichs der Habsburger - neben anderen. Sie war aber anders als die anderen belastet mit einem Namen, der sie in eine Kontinuität mit dem alten Österreich stellte. Das neue Österreich, die Republik, war durch ein besonderes Defizit ausgestattet: mit dem Fehlen einer eigenen, spezifisch österreichischen nationalen Identität.

Diese Besonderheiten begleiteten die Republik: Sie konnte zunächst nicht zu einer eigenständigen Rolle finden. Die herrschenden Kräfte der Republik verstanden, 1918 und danach, das Recht auf Selbstbestimmung als einen Rechtsanspruch auf den „Anschluss“. Und als dieser dann 1938 hereinbrach - in einer Art und Weise, die nicht den Wünschen der Republikgründer, sehr wohl aber den Sehnsüchten eines Teils der österreichischen Gesellschaft entsprach -, zog dieser „Anschluss“ Österreich in die größte Katastrophe Europas hinein.

In den Jahren nach 1945 wurde die Zwischenkriegszeit fast nur in Form einer Rechtfertigungsliteratur wahrgenommen. Die politisch-weltanschaulichen Lager begründeten, dass die Verantwortung für das Scheitern der Republik nach 1918 primär bei der jeweils anderen Seite lag. Die Sozialdemokratie stützte sich auf die umfangreiche Arbeit des US-amerikanischen Historikers Charles Gulick, und die CSP wollte sich mit Berufung auf den Briten Gordon Shepherd vor allem als Gegner des Nationalsozialismus darstellen (vgl. Literaturverzeichnis). Diese Versuche einer Rechtfertigung waren verständlich - denn es gab ja die Kontinuität der politischen Parteien, der politischen Eliten. Aber sie drückten immer nur eine bestimmte, nicht eine allgemeine Sicht der Entwicklung aus.

Diese Parteilichkeit konnte sich erst ändern, als neue Generationen, frei von der Neigung zur Rechtfertigung, diese Zeit zu beschreiben und analysieren begannen. Die Frage nach der „Schuld“ am Versagen der Republik wurde zunehmend durch die Frage nach der „Verantwortung“ ersetzt. Und die Frage nach der Opferrolle Österreichs zwischen 1938 und 1945 machte allmählich einer Unterscheidung zwischen dem Staat Österreich, der ein Opfer des nationalsozialistischen Deutschland war, und der Mitverantwortung weiter Teile der österreichischen Gesellschaft an den Verbrechen des NS-Staats Platz. So konnte auch die internationale, die globale Dimension der Entwicklungen zwischen 1918 und 1938 differenziert wahrgenommen werden.

In den zwei Jahrzehnten zwischen den Weltkriegen sah sich Österreich in einem Umfeld, in dem das 1918 dem Anschein nach so erfolgreiche Prinzip der Demokratie rasch ausgehöhlt wurde. Schon 1933, als Österreichs Regierung den Weg in Richtung eines autoritären Systems beschritt, waren von den Nachbar- 
staaten nur noch die Schweiz und Tschechoslowakei liberale Demokratien. Und der gesamte Raum Zentral- und Mitteleuropas war zum Spielball der Großmachtpolitik des nationalsozialistischen Deutschland und des faschistischen Italien geworden. Mitteleuropa, definiert als der geopolitische Raum, den bis 1918 Österreich-Ungarn eingenommen hatte, war Objekt und nicht Subjekt der Politik.

Das sollte sich 1945 zunächst nicht ändern. Quer durch Mitteleuropa verlief die Front des Kalten Kriegs. Österreich, durch die Zufälligkeit der Geopolitik auf der Westseite dieser Frontlinie, orientierte sich zunehmend an einem Muster, das 1918 kaum beachtet worden war: an der Schweiz und deren Verbindung von Demokratie und Neutralität. Als zwischen 1989 und 1991 der Kalte Krieg zu Ende ging, begann Österreich - letztlich in Übereinstimmung mit den anderen Staaten Mitteleuropas - sich auf die Teilnahme an der europäischen Integration hin zu orientieren. Österreich verstand sich mehr und mehr als Teil Europas. Als Teil der Europäischen Union will und kann Österreich verhindern, wieder Objekt der Expansionspolitik irgendeiner Großmacht zu werden; und als Teil des vereinten Europas sieht Österreich die Chance, die Gespenster von gestern zu bannen - den Nationalismus und Totalitarismus.

Die Voraussetzungen, die Österreich 1945 vorfand, waren andere als 1918. Die Alliierten hatten Österreich - das durch keine Exilregierung vertreten war - in der „Moskauer Deklaration“ 1943 die Selbstständigkeit versprochen. Und angesichts des Schreckens des real erlebten „Anschlusses“ musste Österreich 1945 nicht mehr zur Selbstständigkeit gezwungen werden. Das abermals von Siegermächten definierte Österreich griff schon am 27. April 1945 - dem Tag der „Unabhängigkeitserklärung“ - diese historische Chance auf.

Dass 1945 dieselben Kräfte, teilweise dieselben Personen (allen voran Karl Renner, der Staatskanzler von 1918 und von 1945) die gescheiterte Republik wieder gründeten; dass sie mit größter Selbstverständlichkeit sich auf die Verfassung von 1920 beriefen - das alles zeigt eine Kontinuität. Aber die 1945 wieder gegründete Republik - die „Zweite Republik“ - unterschied sich von der „Ersten“ durch eine politische Kultur des Kompromisses und der Machtteilung. Die politischen Eliten des Lands - und letztlich auch die Gesellschaft insgesamt - hatten aus dem selbst verschuldeten Scheitern gelernt.

Die Staats- und Republikgründer waren 1945 dieselben wie 1918. Doch sie hatten ihr Verhalten geändert. Sie waren weniger „ideologisch“, sie waren nicht mehr die Weltanschauungsparteien der Vergangenheit. Die vormals Christlichsozialen nahmen bewusst nicht mehr das Christliche in ihre Parteibezeichnung auf und demonstrierten so ihre Distanz zum politischen Katholizismus der Vergangenheit. Die Sozialdemokratische Partei nahm Schritt für Schritt Abstand von einem marxistischen Verständnis von Politik und Gesellschaft. Und der „Anschluss“? Der war tot. Der real erlebte „Anschluss“ hatte den Traum vom Aufgehen in ein großes Reich aufgelöst. 


\section{Literatur}

Charles Adams Gulick, Austria. From Habsburg to Hitler, 2 Bde., Berkeley 1948. Anton Pelinka u. a. (Hrsg.), Geschichtsbuch Mitteleuropa. Vom Fin de Siècle bis zur Gegenwart, Wien 2016.

Anton Pelinka, Die gescheiterte Republik. Kultur und Politik in Österreich 19181938, Wien 2017.

Walter Rauscher, Die verzweifelte Republik. Österreich 1918-1922, Wien 2017.

Richard Saage, Der erste Präsident. Karl Renner - eine politische Biografie, Wien 2016.

Gordon Shepherd, Engelbert Dollfuß, Graz 1961.

Gordon Shepherd-Brook, Der Anschluss, Graz 1963.

Peter Thaler, The Ambivalence of Identity. The Austrian Experience of NationBuilding in a Modern Society, West Lafayette 2001. 